\title{
De la description historique d'une hernie de Jean-Louis Petit
}

\section{A seminal description of a Jean-Louis Petit hernia}

\author{
S. Caruba $\cdot$ P. Renucci $\cdot$ S. Chanalet $\cdot$ Y.-E. Claessens $\cdot$ N. Beau
}

Reçu le 16 septembre 2014; accepté le 5 novembre 2014

(C) SFMU et Lavoisier SAS 2014

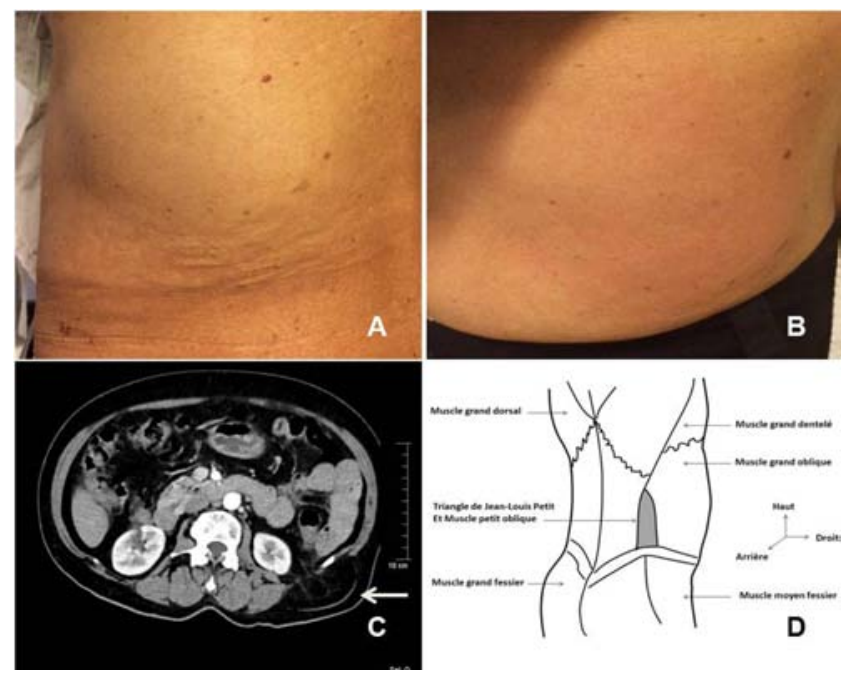

Fig. 1 Tuméfaction douloureuse de la fosse lombaire gauche (A), réductible à la pression (B) chez une patiente de 67 ans sans antécédent. C. Scanner abdominal (acquisition hélicoïdale) après injection de produit de contraste retrouvant une hernie postérieure au niveau du triangle de Jean-Louis Petit (flèche), refoulant et étirant le carré des lombes gauches contenant de la graisse péritonéale et quelques vaisseaux avec attraction de la face postérieure du côlon gauche qui reste intra-abdominale. D. Schéma anatomique décrivant le siège de la hernie de Jean-Louis Petit, consistant en la déhiscence du fascia transversalis et du muscle petit oblique (zone grisée), circonscrit par un triangle défini par le muscle grand oblique en avant, le muscle grand dorsal en arrière, la crête iliaque postérosupérieure en bas
«J'en ai vu une, de la grosseur de la tête d'un enfant, placée entre les fausses côtes de la partie postérieure de la crête de l'os des îles du côté gauche (Fig. 1A); elle disparaissait très souvent lorsque la malade était couchée ; d'autres fois, on était obligé de la presser pour la faire rentrer (Fig. 1B); mais un jour que ni la situation ni la pression n'avaient pu réussir, la malade tomba dans les accidents de l'étranglement, et me fit appeler à son secours. Je trouvais la tumeur beaucoup plus grosse qu'elle n'avait jamais été (du moins selon le rapport que l'on me fit) ; personne ne soupçonnait que ce fût une hernie (Fig. 1C, matériel supplémentaire en ligne). Les uns regardaient cette tumeur comme un dépôt laiteux, d'autres la regardaient comme venteuse. Il est vrai que jusqu'alors elle n'avait été accompagnée d'aucuns des accidents de la hernie, et que d'ailleurs le lieu où elle était placée n'est pas un lieu ordinaire aux hernies ; mais, malgré tout cela, et quoique je n'en eusse jamais vu de cette espèce, les nausées, les défaillances et les vomissements des matières stercorales ne me permirent pas de douter que ce ne fût une vraie hernie qui s'était faite à travers les fibres aponévrotiques du transversal, entre le muscle triangulaire et l'endroit où finissent les obliques (Fig. 1D). » JeanLouis Petit, 1736 [1].

Matériel supplémentaire en ligne Échographie lombaire bidimensionnelle retrouvant une formation herniaire (nodule hypoéchogène extra-abdominal) siège de septa échogènes, mesurant $7 \mathrm{~cm}$ de grand axe et $3 \mathrm{~cm}$ d'épaisseur, dont la mobilisation provoque le passage de tissu graisseux intraabdominal au travers d'une brèche de $15 \mathrm{~mm}$ de grand axe.

\section{Références}

1. Anonyme (1837) Euvres complètes de Jean-Louis Petit. In: Hernie. Académie Royale de Chirurgie, Paris. 695 p

S. Caruba $\cdot$ P. Renucci $\cdot$ S. Chanalet $\cdot$ Y.-E. Claessens $(\triangle)$.

N. Beau

Centre hospitalier Princesse Grace,

département de médicine d'urgence,

1 avenue Pasteur BP 489, MC-98012 Principauté de Monaco

e-mail : yann-erick.claessens@chpg.mc 